Case Report

\title{
Intraosseous myofibroma simulating an odontogenic lesion
}

\author{
Denis P. Souza ${ }^{1,2)}$, Caio C. S. Loureiro ${ }^{2)}$, Roberto A. G. Rejas ${ }^{3)}$, \\ Suzana O. M. Sousa ${ }^{3)}$ and Ricardo Raitz ${ }^{4)}$ \\ ${ }^{1)}$ Department of Oral and Maxillofacial Surgery, School of Dentistry, University of São Paulo, \\ São Paulo, Brazil \\ ${ }^{2)}$ Department of Oral and Maxillofacial Surgery, Hospital Santa Paula, São Paulo, Brazil \\ ${ }^{3)}$ Department of Oral Pathology, School of Dentistry, University of São Paulo, São Paulo, Brazil \\ ${ }^{4)}$ Department of Biodentistry, School of Dentistry, Ibirapuera University, São Paulo, Brazil
}

(Received 25 October 2008 and accepted 13 February 2009)

\begin{abstract}
Myofibroma is a rare benign nodular tumor of the soft tissues, bones, or internal organs, and may affect both children and adults. It is mostly found in the head and neck region, although uncommon in the jaw bones, where only a few lesions have been described. Radiographically, it may appear as a welldefined unilocular radiolucent entity simulating a cystic or odontogenic lesion. Histopathologic findings alone may be insufficient for a final diagnosis, and immunostaining for markers such as vimentin, alphasmooth muscle actin, desmin and S-100 may be required. Intraosseous myofibroma is a diagnostic challenge and requires careful histopathologic, immunohistochemical and radiographic correlation. The present article describes a conservatively treated intraosseous myofibroma that occurred in a 7 -year-old girl. The clinical, radiographic, histopathologic and immunohistochemical features are discussed. (J Oral Sci 51, 307-311, 2009)
\end{abstract}

Keywords: intraosseous myofibroma; radiolucent lesion; radiographic interpretation; immunohistochemistry.

\section{Introduction}

Myofibroma is a rare benign tumor that was originally

Correspondence to Dr. Ricardo Raitz, Rua Heitor Penteado, 1832, 101/A, São Paulo, ZIP Code 05438-300, SP, Brazil

Tel: +55-11-36735270

Fax: +55-11-36735270

E-mail: ricardoraitz@ig.com.br described as a form of congenital multicentric fibroblastic proliferation by Stout in 1954 (1). It affects equally males and females and typically arises in soft tissues with a predilection for the head and neck (2). Internal organs (such as the lungs, kidneys, pancreas, and gastrointestinal tract) have been described as rare sites, presenting single or multiple nodules $(3,4)$. A division has been proposed to differentiate solitary neoplasms and multiple lesions, the latter being termed myofibromatosis (5).

In the oral cavity, some cases involving the mandible, tongue, lips, cheek, maxilla, palate, pterygomandibular raphae, floor of mouth, and submandibular gland have been reported (6-8). However, intraosseous myofibroma, especially within the jaws, is still not widely known or described (7). In such cases, the lesions occur more commonly in children, particularly in the mandible. In adults the development of a solitary intraosseous myofibroma is even rarer $(2,6)$.

The lesion is considered to be completely benign and its extent determines the type of treatment, which can range from a conservative surgical to more aggressive therapies, such as en bloc resection associated with placement of a rigid reconstruction plate and bone grafting $(1,9)$. Unfortunately, some cases are more difficult to diagnose, and thus may be managed with inappropriate therapy. When present in the jaws, the lesions exhibit clinical and radiographic features suggestive of an odontogenic tumor or cyst as well as several other neoplastic conditions (4). Moreover, histopathologically, there is a great potential for confusion with the more aggressive spindle cell tumor (2).

Here we present the clinical, radiographic, histo- 
pathologic and immunohistochemical findings in a challenging case of central myofibroma in an attempt to draw attention to the difficulties in properly diagnosing this lesion.

\section{Case Report}

A 7-year-old girl was referred to our private stomatology and maxillofacial surgery office because her orthodontist had found a radiolucent lesion at the base of the right mandible. The patient had no symptoms and her medical and family history were non-contributory.

Physical examination revealed an elastic, firm, localized, tenuous, very discrete swelling that involved the right mandibular vestibule. Panoramic radiography demonstrated a well defined unilocular radiolucent lesion in the right mandibular body, with corticated borders, beneath the mental foramen. The lesion was located beneath the second premolar, which was still in the process of formation. A computed tomography (CT) cone beam was taken to evaluate the extent of the lesion, and this revealed a $2-\mathrm{cm}$ well demarcated, expansive, ovoid low-density area in the right mandibular body with partial resorption of the buccal cortical bone. The line of the mandibular canal was preserved, as well as the vestibular plate. These findings suggested a diagnosis of odontogenic keratocystic tumor (Figs. 1 and 2).

The patient was admitted to our hospital for surgical intervention. Under general anesthesia, an extra-oral approach was taken to reach the lesion in order to avoid any damage to the mental nerve. Osteotomy of the mandibular cortical bone was performed using round burs and irrigation at low-speed rotation. Contrary to our expectation of a cystic lesion, an ovoid intraosseous, yellow-colored, non-bleeding solid tumor was found. During exploration of the lesion site, the mass was easily detached from the surrounding mandibular bone, and

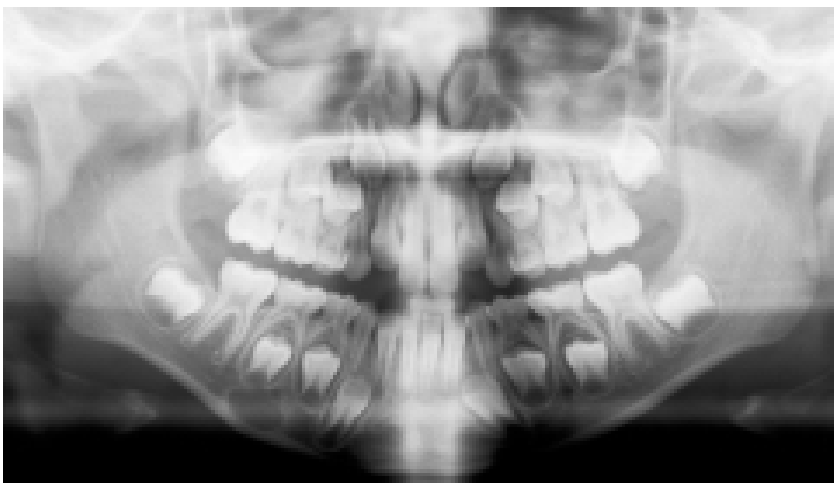

Fig. 1 Unilocular radiolucent lesion, with defined limits and a tenuous radiopaque halo, next to the apex of the 45 tooth germen.

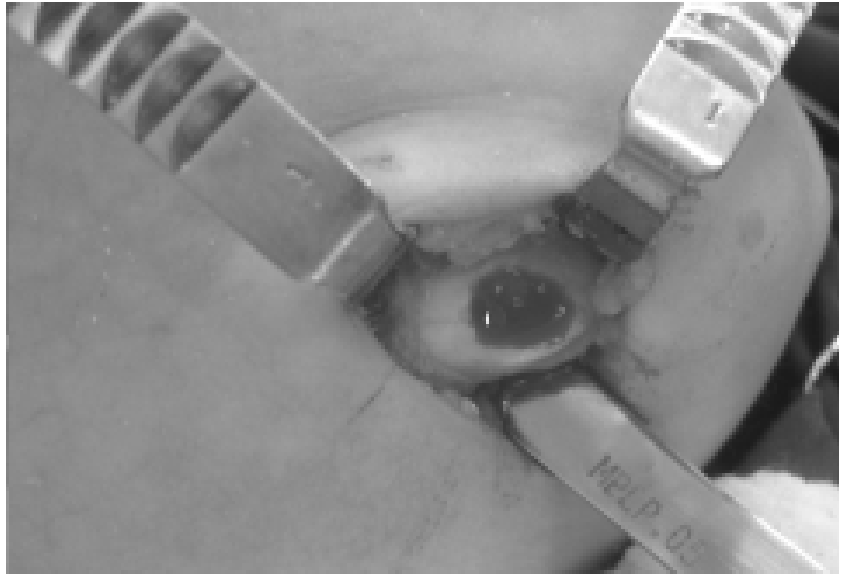

Fig. 3 Extra-oral access: enucleation and vigorous curettage.

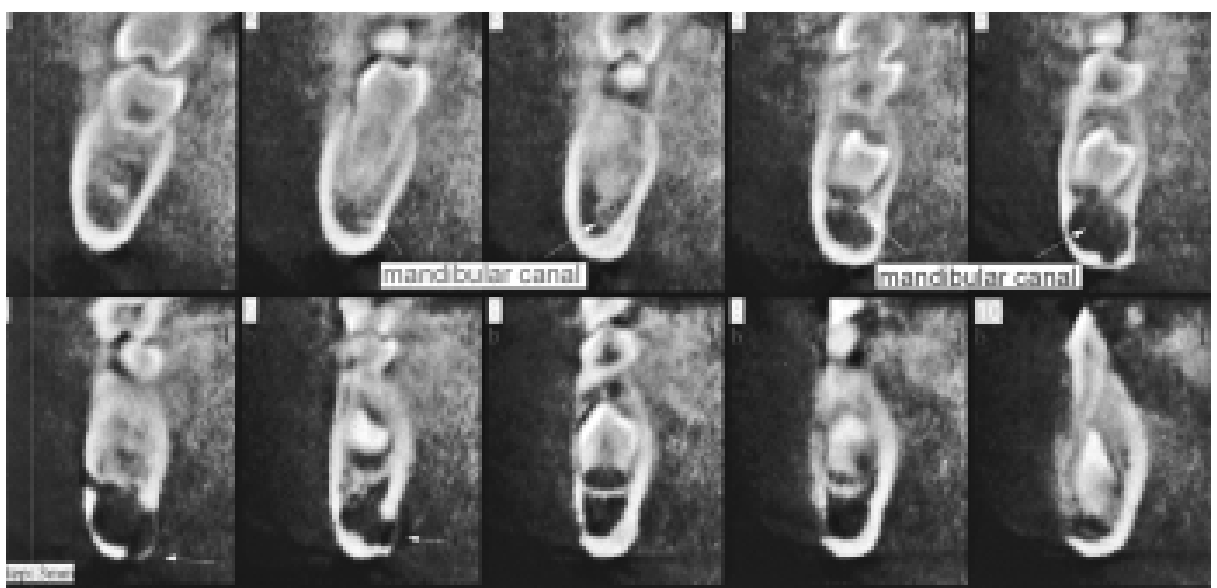

Fig. 2 Cone beam computed tomography showing a unique locus with absorption of the lingual bone plate (arrows at lower column). 
therefore we decided to perform an excisional biopsy and curettage (Fig. 3). The compressed second premolar was preserved.

Histopathological examination of the specimen revealed richly cellular, haphazardly arranged, interweaving bundles of short or long fascicles of spindle-shaped cells. Individually, the spindle cells had oval nuclei with tapered or rounded ends and occasional nuclear grooves. Some areas showed a hemangiopericytoma-like pattern, with multiple slit-like vascular spaces. No mitotic figures were observed (Figs. 4 and 5).

Immunohistochemical staining was positive for vimentin, muscle-specific actin (HHF35) and smooth muscle actin (SMA) (Figs. 6 and 7). No immunoreactivity for S-100,

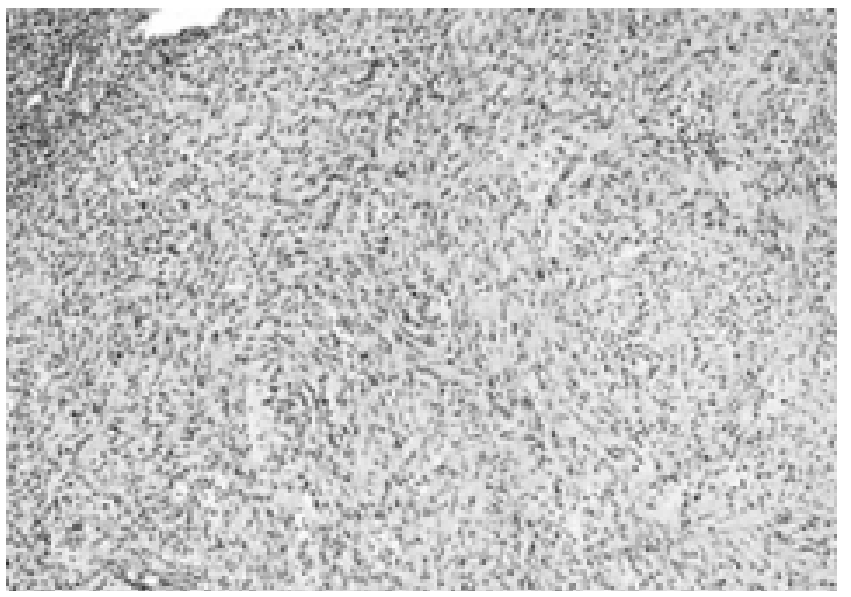

Fig. 4 Panoramic view of the myofibroma showing a biphasic pattern composed of cells with small, round nuclei and spindle cells forming short fascicles or whorls (H-E staining, original magnification $\times 25$ ).

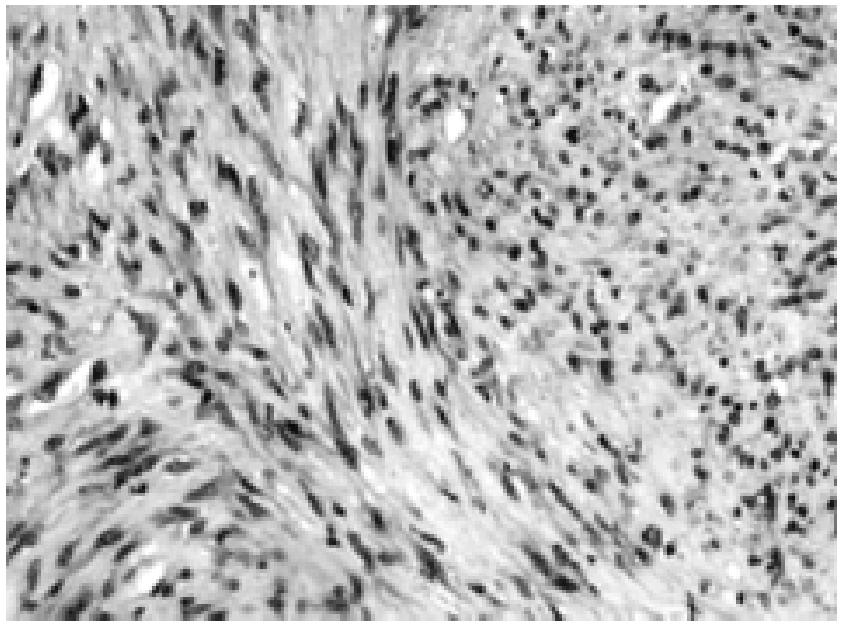

Fig. 5 Spindle cells are arranged haphazardly in short fascicles (H-E staining, original magnification $\times 200$ ). desmin or enolase (NSE) was observed.

Medical and radiographic evaluation confirmed the solitary aspect of the lesion, thus excluding myofibromatosis. The patient is still receiving treatment for postoperative control (Fig. 8). At the 3-year follow up, there were no signs of lesion recurrence, and the bone had completely reformed.

This study was performed in accordance with the ethical standards laid down in the 1964 Declaration of Helsinki, and the child's guardian gave informed consent to all examinations and treatments.

\section{Discussion}

Myofibroma is a rare benign tumor presenting as a

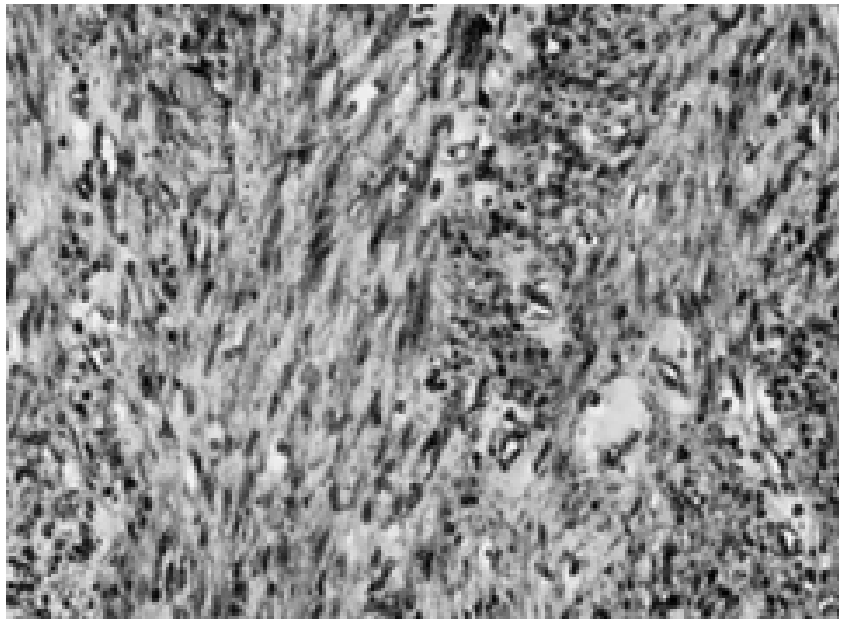

Fig. 6 Vimentin is expressed by most of the tumoral cells (dark black). Streptavidin-biotin staining, $\times 200$.

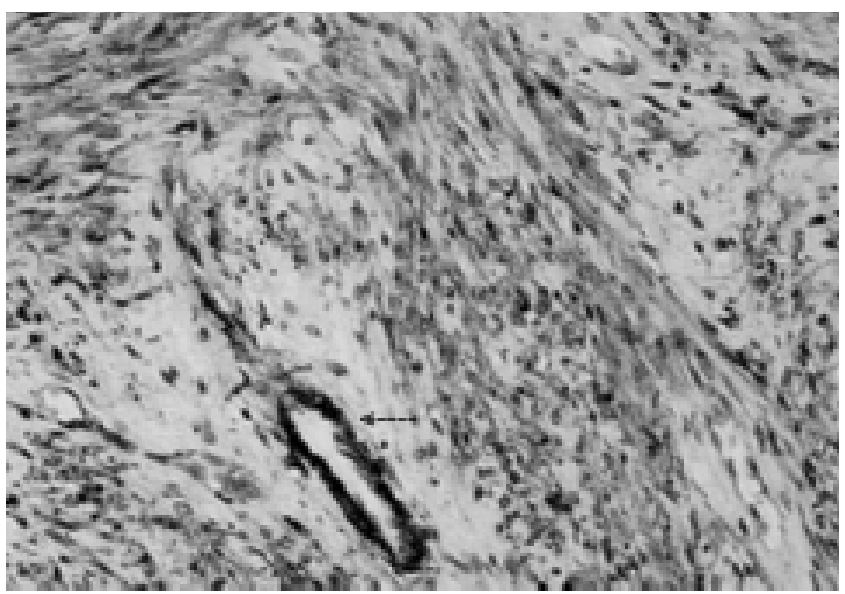

Fig. 7 Smooth muscle actin expression is evident in many of the tumor cells (dark black). Note that the vessel wall is also positive (arrow). Streptavidin-biotin staining, $\times 200$. 


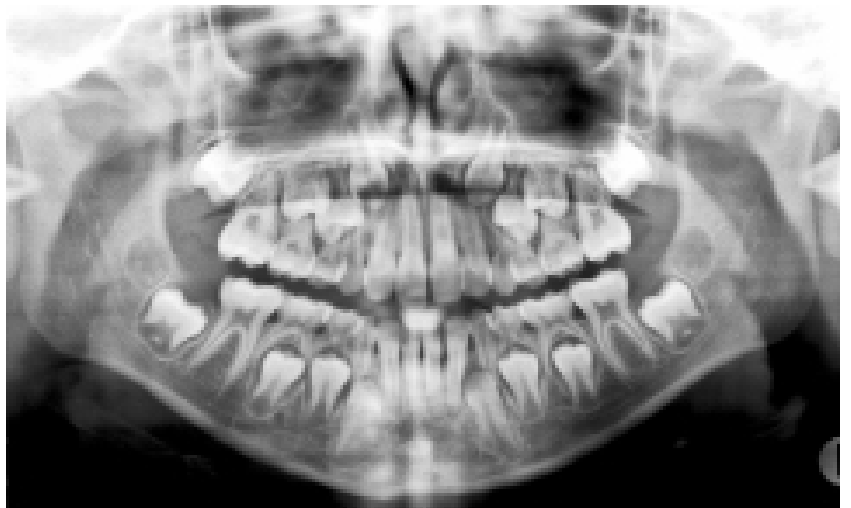

Fig. 8 Local osteogenesis 10 months after excision.

solitary mass or multiple nodular lesions, the prognosis depending on the lesion location. According to Kauffman and Stout (10), this lesion can be divided into two types depending on its anatomical localization: those with a good prognosis affect the skin, subcutaneous tissue, or skeleton, and those with a poor prognosis affect the soft tissue, muscles, bone or internal organs. In the present case, conservative treatment was possible because of good cooperation among the professionals responsible, including a radiologist, a stomatologist, a pathologist and an oral surgeon.

The cause of myofibroma is currently unknown. Some authors suggest that the tumor is inherited as an autosomal dominant or autosomal recessive trait, although very low familial incidence has been reported $(10,11)$. As the tumor presents as a slow-growing, nodular, firm submucosal swelling, it may be difficult to diagnose $(5,11)$. In the present case, the lesion was not evident by intra-oral palpation and did not cause asymmetry, but was detected by orthodontic panoramic radiography, confirming that this imaging method is important for evaluating the lesion.

Radiographically, this case of intraosseous myofibroma was imaged as a radiolucent unilocular lesion with a well defined border. Among previously reported intraosseous cases, $67 \%$ have shown the same characteristics (3), and around $30 \%$ have shown a multilocular appearance. Occasionally, there may be evidence of cortical expansion and/or perforation, which in the present case was seen only on CT examination $(3,4)$. We emphasize that an apparently innocuous lesion in children, which has a cystic appearance on radiographs, must always be investigated with accurate imaging techniques.

Histopathologically, intraosseous myofibroma must be carefully distinguished from other types of fibromatosis, and this may not be easy. Definitive histologic diagnosis is particularly difficult because of its similarity to other spindle-cell lesions. Differential diagnosis of spindle cell proliferations should include both benign lesions, such as those of nerve tissue origin, or leiomyoma, and malignant lesions such as congenital fibromatosis, fibromatosis, lowgrade fibrosarcoma and leiomyosarcoma (12-16). A definitive diagnosis of intraosseous myofibroma is generally established on the basis of light microscopy, but immunohistochemical findings as well as electron microscopy are certainly useful for confirming the diagnosis. Spindle cell lesions of nerve tissue origin can be excluded because of their immunoreativity for S-100, which is absent in myofibroma. Leiomyoma and leiomyosarcoma of bone are rare, and can be excluded on the basis of their immunoreactivity for desmin, which is negative in myofibroma, and also by the histologic appearance of leiomyoma, which consists of cells arranged into long fascicles intersecting at right angles $(8,17)$.

Myofibroma must be carefully distinguished from the significantly more destructive and aggressive fibromatosis (6). Histologically, fibromatosis has a more monophasic growth pattern comprising long sweeping fascicles of spindle cells among abundant wavy collagen fibrils, which are not a feature of myofibroma (6). Fibrosarcoma of the bone can be differentiated by the presence of a "herring bone" pattern, nuclear atypia and high mitotic counts including abnormal mitoses (18). Therefore, careful microscopic and immunohistochemical examinations allow the separation of intraosseous myofibroma from other tumors of the oral cavity. In the present case, immunohistochemical staining was decisive for establishing the correct diagnosis, as reported previously for other cases $(8,13,16,19)$.

Conservative treatment is the choice for $75 \%$ of patients with intraosseous myofibroma (3). In the present case, the small extent and easy detachment of the lesion from the mandibular bone allowed conservative surgical excision with preservation of the tooth. The outcome after a 2-year follow-up is excellent. Such treatment, preserving all anatomical, functional and esthetic structures, could only have been possible with an accurate diagnosis based on a combination of radiographic, histopathologic and immunohistochemical evidence, and cooperation among different specialists.

In conclusion, careful radiographic, microscopic and immunohistochemical examinations are necessary for proper diagnosis of intraosseous myofibroma, in order to distinguish it from cystic lesions and other neoplasms of the oral cavity. 


\section{References}

1. Stout AP (1954) Juvenile fibromatosis. Cancer 7, 953-978.

2. Oliver RJ, Coulthard P, Carre C, Sloan P (2003) Solitary adult myofibroma of the mandible simulating an odontogenic cyst. Oral Oncology 39, 626-629.

3. Allon I, Vered M, Buchner A, Dayan D (2007) Central (intraosseous) myofibroma of the mandible: clinical, radiologic, and histopathologic features of a rare lesion. Oral Surg Oral Med Oral Pathol Oral Radiol Endod 103, e45-e53.

4. Sedghizadeh, PP, Allen CM, Kalmar JR, Miloro M, Suster S (2004) Solitary central myofibroma presenting in the gnathic region. Ann Diagn Pathol 8, 284-289.

5. Lingen MW, Mostofi RS, Solt DB (1995) Myofibromas of the oral cavity. Oral Surg Oral Med Oral Pathol Oral Radiol Endod 80, 297-302.

6. Foss RD, Ellis GL (2000) Myofibromas and myofibromatosis of the oral region: a clinicopathologic analysis of 79 cases. Oral Surg Oral Med Oral Pathol Oral Radiol Endod 89, 57-65.

7. Montgomery E, Speight PM, Fisher C (2000) Myofibromas presenting in the oral cavity: a series of 9 cases. Oral Surg Oral Med Oral Pathol Oral Radiol Endod 89, 343-348.

8. Sugatani T, Inui M, Tagawa T, Seki Y, Mori A, Yoneda J (1995) Myofibroma of the mandible. Clinicopathologic study and review of the literature. Oral Surg Oral Med Oral Pathol Oral Radiol Endod 80, 303-309.

9. Troulis MJ, Williams WB, Kaban LB (2004) Staged protocol for resection, skeletal reconstruction, and oral rehabilitation of children with jaw tumors. J Oral Maxillofac Surg 62, 335-343.

10. Kauffman SL, Stout AP (1965) Congenital mesenchymal tumors. Cancer 18, 460-476.

11. Liu CJ, Chang KW (2001) "Infantile" myofibroma of the oral cavity: report of case. J Oral Maxillofac Surg 59, 471-472.

12. Haedicke G, Kaban LB (1988) Smooth-muscle tumors of the oral cavity. Plast Reconstr Surg 81, 264-269.

13. Hartig G, Koopmann CJr, Esclamado R (1993) Infantile myofibromatosis: a commonly misdiagnosed entity. Otolaryngol Head Neck Surg 109, 753-757.

14. Inwards CY, Unni KK, Beabout JW, Shives TC (1991) Solitary congenital fibromatosis (infantile myofibromatosis) of bone. Am J Surg Pathol 15, 935941.

15. Mechlin DC, Hamasaki CK, Moore JR, Davis WE, Templer J (1980) Leiomyoma of the maxilla-report of a case. Laryngoscope 90, 1230-1233.

16. Raffaini M, Baggi MT, Bozzetti A, Sesenna E, Gabrielli M (1990) Mandibular leiomyoma in an infant. Report of a case. Int J Oral Maxillofac Surg 19, 367-369.

17. Rodini CO, Corrêa Pontes FS, Rebelo Pontes HA, da Silva Santos PS, Gallottini Magalhães M, Santos Pinto DJr (2007) Oral leiomyosarcomas: report of two cases with immunohistochemical profile. Oral Surg Oral Med Oral Pathol Oral Radiol Endod 104, e50-e55.

18. Kahn LB, Vigorata V (2002) Fibrosarcoma. In: World Health Organization classification of tumors. Pathology and genetics of tumours of soft tissue and bone. Fletcher CDM, Unni KK, Mertens F eds, IARC Press, Lyon, 289.

19. Slootweg PJ, Muller H (1984) Localized infantile myofibromatosis. Report of a case originating in the mandible. J Maxillofac Surg 12, 86-89. 